\title{
Arrhythmogenesis in the Short-QT Syndrome Associated With Combined HERG Channel Gating Defects
}

A Simulation Study

\author{
Hideki Itoh, MD*,**; Minoru Horie, MD**; \\ Makoto Ito, MD**; Keiji Imoto, MD*
}

\begin{abstract}
Background This study aimed to show the mechanism how the HERG channel gating defects causes lifethreatening arrhythmia in the short-QT syndrome, using a simulation model of ventricular action potentials (APs). Methods and Results To evaluate the electrophysiological consequences of the short-QT syndrome at the level of the cardiac AP, the Markov model of wild-type (WT) KCNH2 channel was modified to obtain a model of the KCNH2 channel with the N588K mutation associated with the short-QT syndrome. Two parameters ( $\beta \mathrm{i}$ and $\beta \beta$ ) were changed to reconstruct the N588K mutant Markov model, which successfully reproduced the experimental results of voltage-clamp recordings. The WT and mutant models were then integrated into the LuoRudy theoretical model of the cardiac ventricular AP. Unexpectedly, 1 parameter change alone, which caused gain of function, could shorten the AP duration (APD) but failed to induce early after-depolarizations (EADs). Only the condition with the combined gating defects could lead to EAD.

Conclusions Although the gain of function for KCNH2 shortened APD in the short-QT syndrome, this simulation study suggested that arrhythmogenesis was associated not only with gain of function, but also with accelerated deactivation of KCNH2. (Circ J 2006; 70: 502-508)
\end{abstract}

Key Words: HERG; Short QT syndrome; Simulation

$\mathbf{T}$ he short-QT syndrome constitutes a new primary electrical abnormality associated with sudden cardiac death!,2 A part of the disorder has been linked to gain of function mutations in the cardiac channel of rapidly activating delayed rectifier currents (IKr), HERG (KCNH2)? Heterogeneous abbreviation of the action potential duration (APD) in different cell types spanning the ventricular wall seems to create the substrate for the genesis of ventricular arrhythmia in the short-QT syndrome, as is proposed for the long-QT syndrome and Brugada syndrome ${ }^{4-8}$

Brugada et al identified the first mutation associated with the short-QT syndrome? The missense mutation (N588K) involving a substitution of lysine for asparagine in position 588 of KCNH2 was found to cause a remarkable gain of function in the IKr. We studied how the gain of function mutation $\mathrm{N} 588 \mathrm{~K}$ in the KCNH2 could affect APD in different cell types of the ventricular walls, and how it could be associated with life-threatening arrhythmia in the LuoRudy (LRd) theoretical model of the cardiac ventricular action potential (AP) $4,5,7,9$ Unexpectedly, although the gain of function of $\mathrm{KCNH} 2$ resulted in shortening of the APD, arrhythmogenesis was associated not only with the gain of function, but also with accelerated deactivation of the N588K HERG channel.

(Received October 24, 2005; revised manuscript received January 5, 2006; accepted January 27, 2006)

*Department of Information Physiology, National Institute for Physiological Sciences, Okazaki, **Department of Cardiovasclar and Respiratory Medicine, Shiga University of Medical Science, Otsu, Japan Mailing address: Keiji Imoto, MD, Department of Information Physiology, National Institute for Physiological Sciences, Myodaiji, Okazaki 444-8787, Japan. E-mail: keiji@nips.ac.jp

\section{Methods}

To evaluate the electrophysiological consequences of the N588K mutation associated with the short-QT syndrome at the level of the cardiac AP, we constructed a Markov model of the N588K mutant channel, based on the experimental data of voltage-clamp recordings of IKr heterologously expressed in TSA 201 cells? The wild-type (WT) and N588K Markov models were then integrated into the LRd theoretical model of the cardiac ventricular AP5 The simulation programs were encoded in $\mathrm{C}++$ and implemented on a personal computer running Windows XP or an HP AlphaServer running Tru64 UNIX.

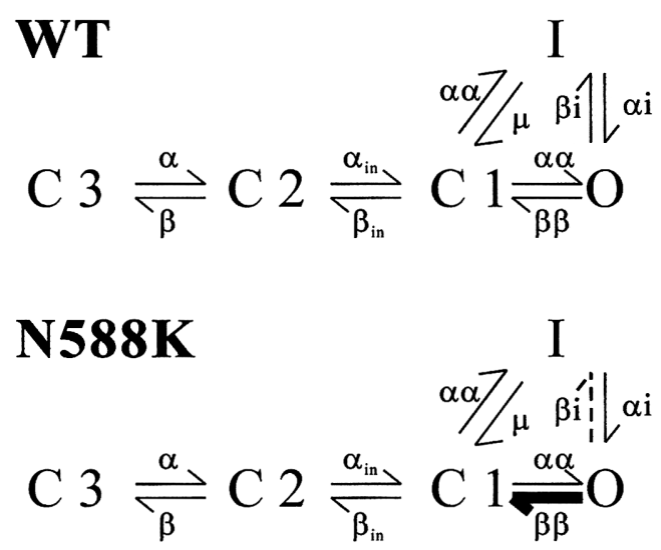

Fig 1. Schemes of the Markov models for wild-type (WT) and N588K IKr channels5. The dotted line shows a decreased rate and the bold line shows an increased rate. 
The Markovian Model of the Cardiac IKr

As a prototype channel model, we used the previously published Markov model of the cardiac IKr channel (appendix of reference 4). Hodgkin-Huxley type models do not represent important properties associated with structurefunction relationships of individual channels, although the Markov scheme represents distinct channel states and coupling between these states, allowing us to relate statespecific kinetic properties of ion channels to the electrophysiological behavior of the whole cell?

The Markov models of the WT and N588K mutant channels are shown in Fig 1 . The model includes 3 closed states $(\mathrm{C} 3, \mathrm{C} 2, \mathrm{C} 1)$, an open state $(\mathrm{O})$, and an inactivation state (I). The transition between $\mathrm{C} 2$ and $\mathrm{C} 1$ is voltage-independent. We modified the basic Markov model as little as possible to simulate the experimental results (Table 1)? The simulation for voltage-clamp experiments was calculated using the fourth-order Runge-Kutta method with a fixed time step of $0.020 \mathrm{~ms}$.

\section{AP Simulations}

We used the dynamic LRd model of a ventricular cell with recent modifications? Simulations were conducted for isolated epicardial, endocardial, and $\mathrm{M}$ cells, which were simulated by varying the maximum conductance of the slowly activating delayed-rectifier potassium current (IKs)?
Table 1 IKr Formulation

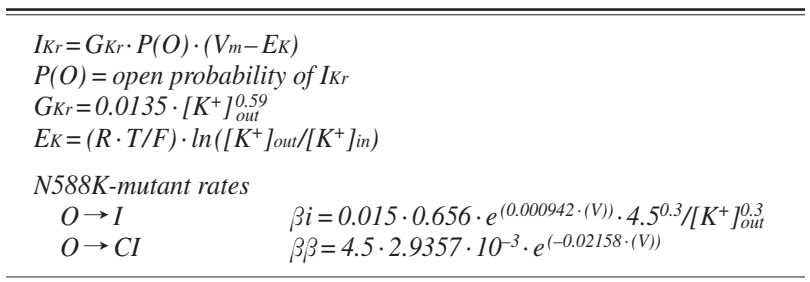

We labeled the simulated cell types with density ratios GKs : GKr $=23: 1,15: 1$, and 7:1 as epicardial cells, endocardial cells, and M cells, respectively? The absolute value of the maximal repolarization time difference was considered as the maximal transmural dispersion of repolarization (TDRmax). All cells were paced over 1,000 times at any basic cycle lengths (BCL). The APD90 (APD50) was defined as APD at $90 \%(50 \%)$ repolarization.

The AP simulation was calculated using the method by Rudy et al ${ }^{10}$ (http://www.cwru.edu/med/CBRTC/LRdOnline/ development.htm) with a flexible time step ranging from $0.002 \mathrm{~ms}$ to $0.100 \mathrm{~ms}$.
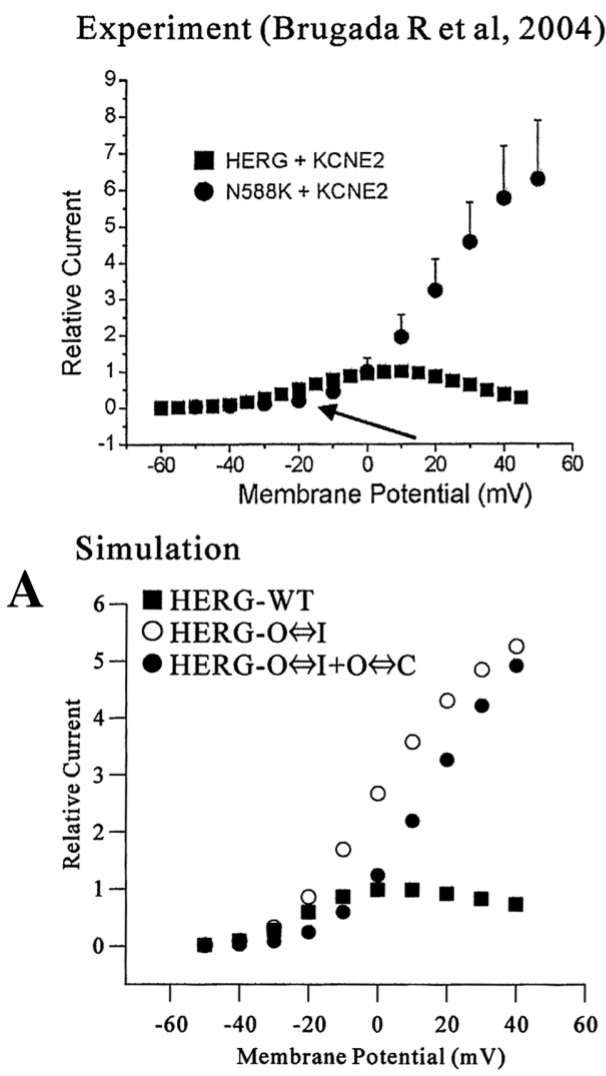
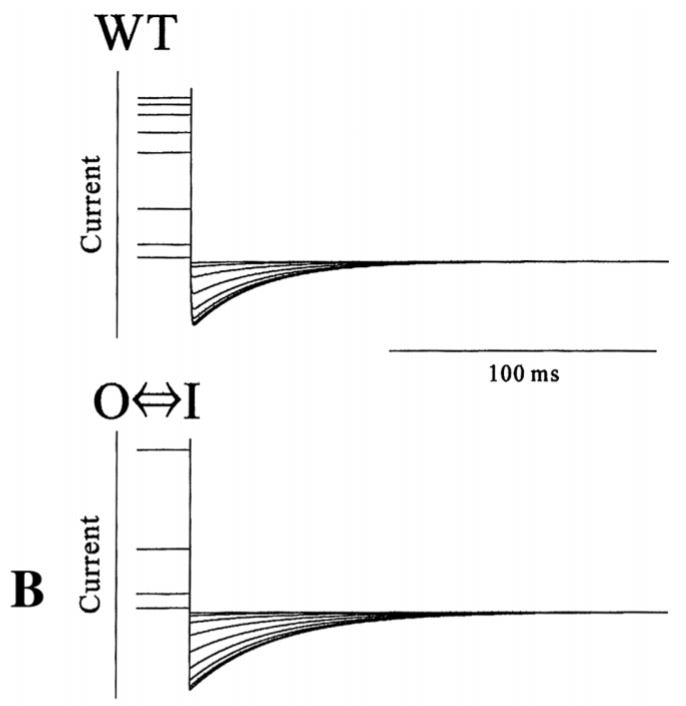

$\mathrm{O} \Leftrightarrow \mathrm{I}+\mathrm{O} \Leftrightarrow \mathrm{C}$

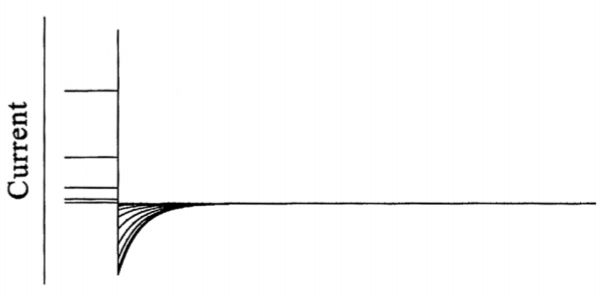

Fig 2. The experimental and simulation results for voltage-clamp protocols. (A) The peak current-voltage relationship. The simulated current-voltage relationship showed that the change of $\beta i$ alone failed to rectify significantly within a physiological range of potentials as the experimental study, because the current of N588K mutant should be smaller than that of wild-type (WT) at range below $0 \mathrm{mV}$ (arrow). By changing $\beta \beta$ in addition to bi, however, the N588K channel showed smaller currents at voltage clamps below $0 \mathrm{mV}$. (B) The tail current after repolarization. Although the voltage-clamp recordings of $\mathrm{N} 588 \mathrm{~K}$ showed significantly reduced tail currents after repolarization, we could not reproduce the mutant effect by only changing $\beta$ i. The N588K channel showed reduced tail currents by additionally changing $\beta \beta$. The parameters used for simulation were the same as in Clancy and Rudy5 except for $\beta$ i and $\beta \beta$. 

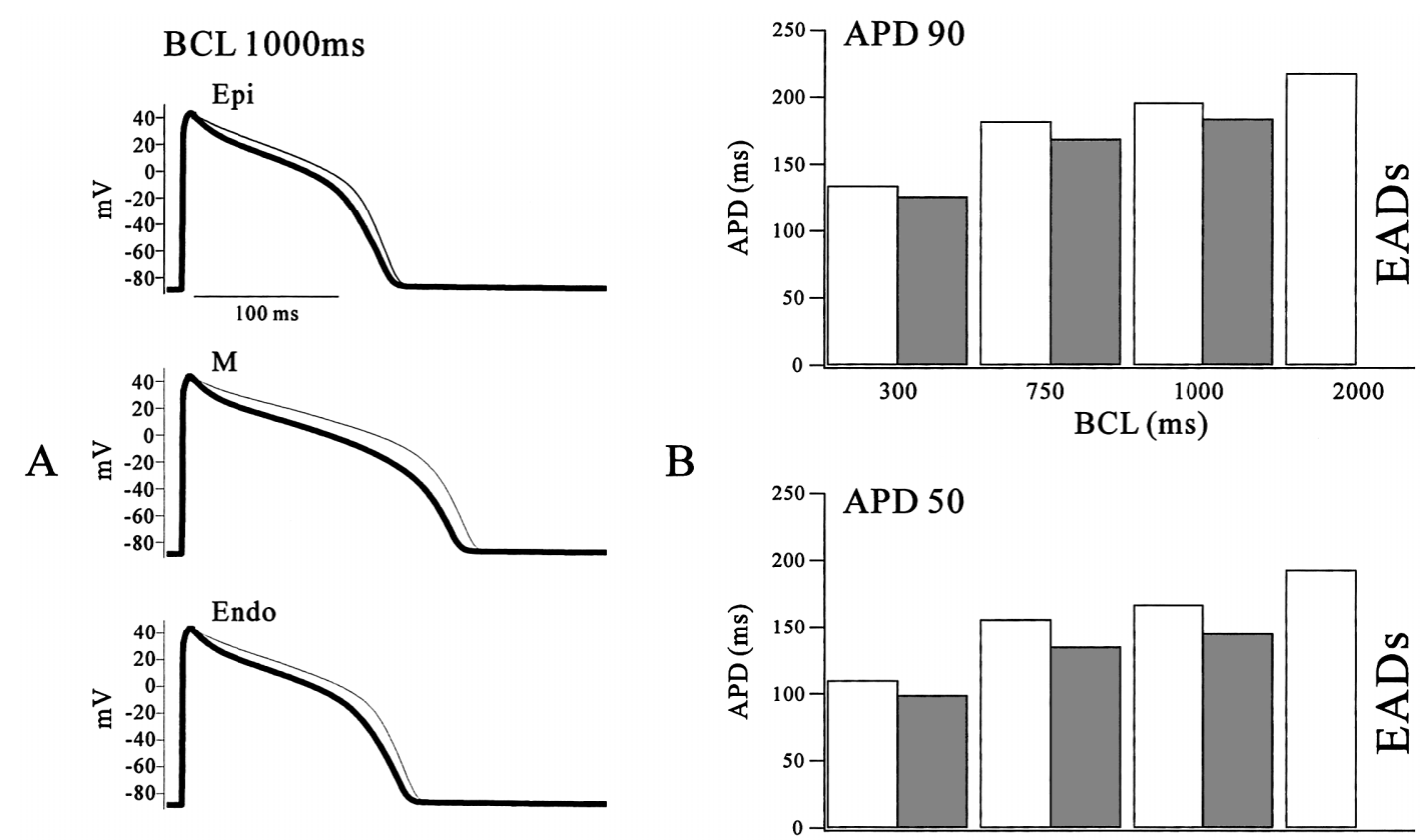

B

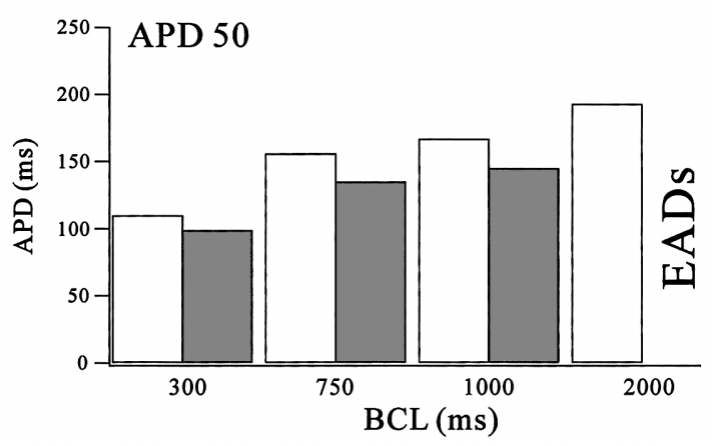

Fig 3. (A) Comparison of action potentials (AP) of wild-type (WT) and N588K on epicardial (Epi), M, and endocardial (Endo) model cells (basic cycle lengths $(\mathrm{BCL})=1,000 \mathrm{~ms}$ ). When the IKr channels were all N588K mutants (thick lines), AP duration (APD) of each wall layers were smaller than WT (thin lines). (B) The frequency dependence of APD in M cells. The APD of N588K (black bar) were shorter than those of WT (white bar) at any BCL tested. Early after-depolarizations (EADs) occurred in the N588K M cell layer at BCL of 2,000 ms.

\section{Results}

\section{The Markov Models for $\mathrm{KCNH} 2$}

The N588K mutation, a point mutation in the S5-pore segment of $\mathrm{KCNH} 2$, dramatically increases IKr? Based on the experimental results of voltage-clamp recordings, we looked for parameters responsible for the gating defects (Table 1)? First, simulation study revealed that the steadystate current continued to increase linearly at membrane potentials by decreasing the transition rate from $\mathrm{O}$ to $\mathrm{I}(\beta \mathrm{i})$. The current-voltage relationships showed that the N588K model failed to rectify significantly within a physiological range of potentials, similarly as in the experimental study (Fig 2A). A change of di also showed the same result as $\beta$ i. The changing rates of other gating parameters could not increase IKr currents (data not shown). These results also agree with other experimental data, which show that N588K channels do not inactivate!1,12 Second, although the N588K channel showed small and fast inactivating tail currents after repolarization, and smaller currents at lower membrane potential (Fig 2A, arrow), we could not reconstitute such tail currents by only changing $\beta$ i. However, by increasing the transition rate from $\mathrm{O}$ to $\mathrm{C} 1(\beta \beta)$, the tail currents of the mutant channel became smaller and deactivated faster (Fig 2B). The changes of gating parameters other than $\beta \beta$ could not accelerate the deactivation of IKr currents (data not shown). The simulated N588K showed the acceleration of the rates of activation as compared with the experimental data. Zhou et al reported that high temperature accelerated the rates of activation ${ }^{13}$ Therefore, we could reasonably well simulate the experimental results of the N588K mutant channel by changing the 2 parameters $\beta \mathrm{i}$ and $\beta \beta$, because the simulation data were performed at physiological temperature, whereas experimental data were performed at room temperature.

McPate et al recently showed no significant difference in deactivation time-course between WT and N588K channels at physiological temperature ${ }^{12}$ These results differ from the reports of Brugada et $\mathrm{al}^{3}$ or Cordeiro et al ${ }^{11}$ First, these results might be associated with experimental conditions, in which McPate et al ${ }^{11}$ studied not only at physiological temperature, but without MiRP1. Second, the balance of transient rates, which are associated with deactivation and inactivation, could determine the scale and time-course of the tail currents of IKr currents. Therefore, the changing of 2 parameters $\beta \mathrm{i}$ and $\beta \beta$ are reasonable, because decelerated transient rate to inactivation could increase the tail currents of IKr currents, whereas accelerated transient rate to deactivation could affect oppositely. The N588K channels with decelerated transient rate to inactivation, but not significant changes of the tail currents of IKr currents, necessarily need to have accelerated deactivation at a physiological temperature.

\section{AP Simulations for LRd Model}

To study the cellular consequences of the mutation, the Markov models of WT and N588K channels were inserted into the LRd model of cardiac ventricular cells.,5 The effects of the N588K mutation on AP of epicardial, endocardial, and $\mathrm{M}$ cells were studied for the BCL of $1,000 \mathrm{~ms}$. When the IKr channel was all N588K mutant, the APD of every wall layers were shorter than WT (Fig 3A). The APD of N588K was shorter than WT at any BCL (Fig 3B).

The IKr currents of WT recorded during AP stimulus displayed a hump-like waveform (Fig 4A). In contrast, N588K currents displayed a dome-like waveform and have a more 


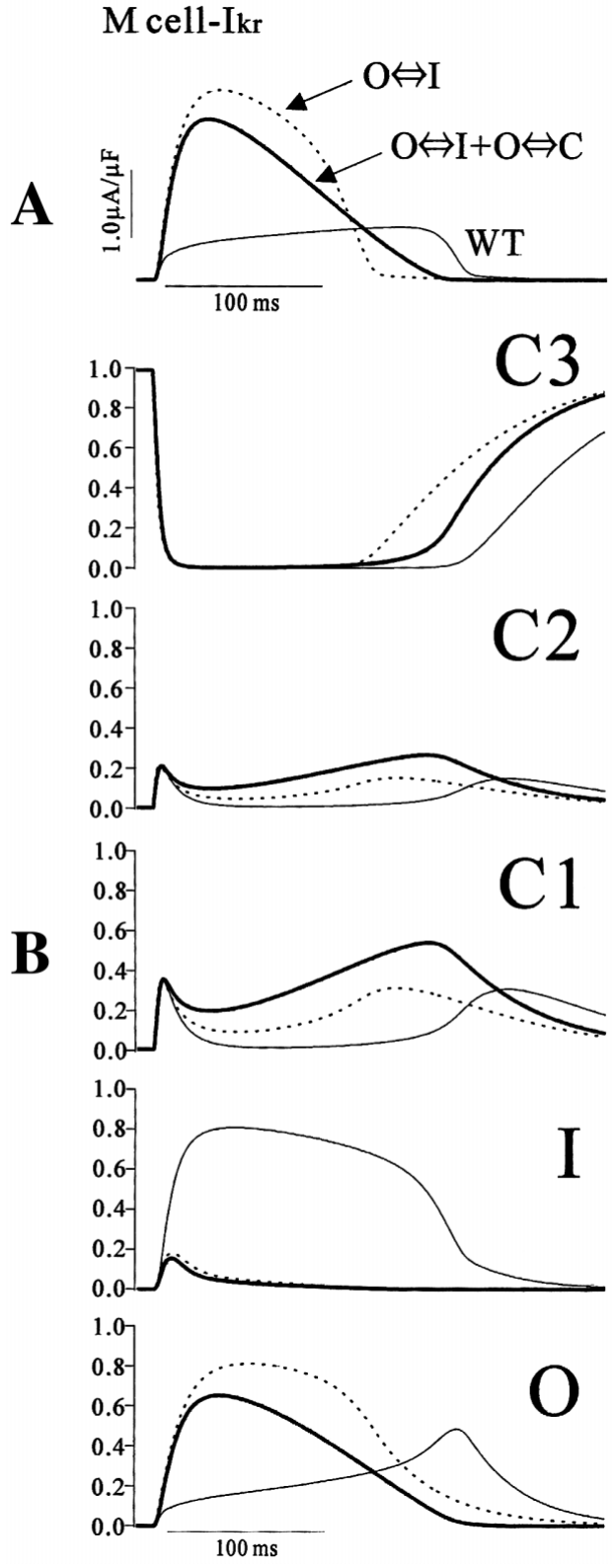

Fig 4. IKr of M cell models of wild-type (WT) and N588K. (A) The IKr currents of WT during action potential (AP) displayed a hump-like waveform. In contrast, N588K currents displayed a dome-like waveform. The change of $\beta \mathrm{i}$ alone caused larger IKr currents than the changes of $\beta$ i plus $\beta \beta$. (B) The probabilities of residence in the indicated states over the course of AP. IKr for N588K opens greater because of the decreased open state to inactivation state (O-to-I) transition. Although the WT channel slowly deactivates (from $\mathrm{O}$ to $\mathrm{C} 1$ transition), the mutant channel deactivates faster than WT. Predictably, the probability on the closed states (is larger) with the changes of $\beta \beta$ plus $\beta$ i showed is larger than with the change of $\beta$ i alone. WT, thin lines; only changing $\beta \mathrm{i}$, dotted lines; changing $\beta \beta$ in addition to $\beta \mathrm{i}$, thick lines.

important contribution during the early phases of repolarization of the AP. These simulated IKr currents showed the same results as experimental studies? 3 In the WT condition, the channel moves from the leftmost closed state (C3) and passes through $\mathrm{C} 2$ and $\mathrm{C} 1$ during the $\mathrm{AP}$ upstroke. From the $\mathrm{C} 1$ state, the channel can pass directly to the $\mathrm{O}$ state or to the I state. Once the channel opens, it rapidly inactivates. The equilibrium is reached between the $\mathrm{O}$ state and the I

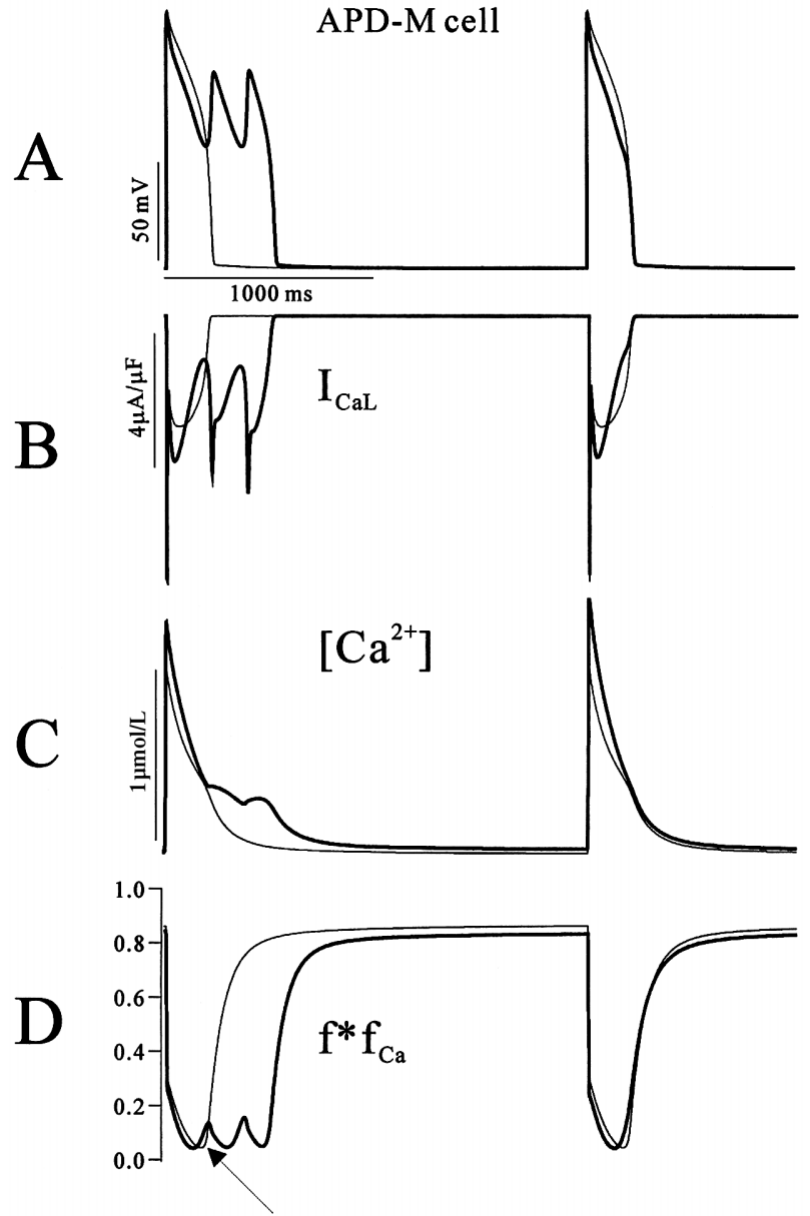

Fig 5. Ionic mechanism of early after-depolarizations in the shortQT syndrome. (A) Action potentials. (B) ICa(L). (C) Calcium transient. (D) A parameter of calcium channel availability, $\mathrm{f} \times \mathrm{fCa}$. The larger calcium transient $(\mathrm{C})$ results in a greater initial inactivation of $\mathrm{ICa}(\mathrm{L})$ (B,D). Wild-type, thin lines; N588K, thick lines. Arrow, the faster recovery from inactivation in the short-QT syndrome allows for the reactivation of $\mathrm{ICa}(\mathrm{L})$.

state, and it begins to favor recovery as the AP plateau repolarizes. As recovery from inactivation becomes more prominent, IKr increases and accelerate late repolarization of the AP4 In case of the N588K mutant channel, however, $\mathrm{IKr}$ appears earlier and is larger because of a decreased probability of the O-to-I transition. Although the WT channel slowly deactivates (from $\mathrm{O}$ to $\mathrm{C} 1$ transition), the mutant channel deactivates faster than WT (Fig 4B). Predictably, changing $\beta \mathrm{i}$ alone caused larger IKr currents than changing $\beta \beta$ plus $\beta \mathrm{i}$ (Fig $4 \mathrm{~A}$ ), and the probability of populating the closed states is larger with the changes of $\beta \beta$ plus $\beta$ i than with the change of $\beta$ i alone (Fig 4B).

\section{Early After-Depolarizations (EADs) of the Short-QT Syndrome}

Although the N588K mutant channel has decreased APD, EADs occur with the BCL of $2,000 \mathrm{~ms}$ in $\mathrm{M}$ cells, but not in epicardial or endocardial cells (Fig 5A). The inward currents mediated by the L-type Ca channel (ICa(L)) are a critical determinant of AP and also of EADs. In association with the N588K mutant channel, they show a more prominent dome and greater recovery from inactivation at a membrane potential in the range of $\mathrm{ICa}(\mathrm{L})$ activation 

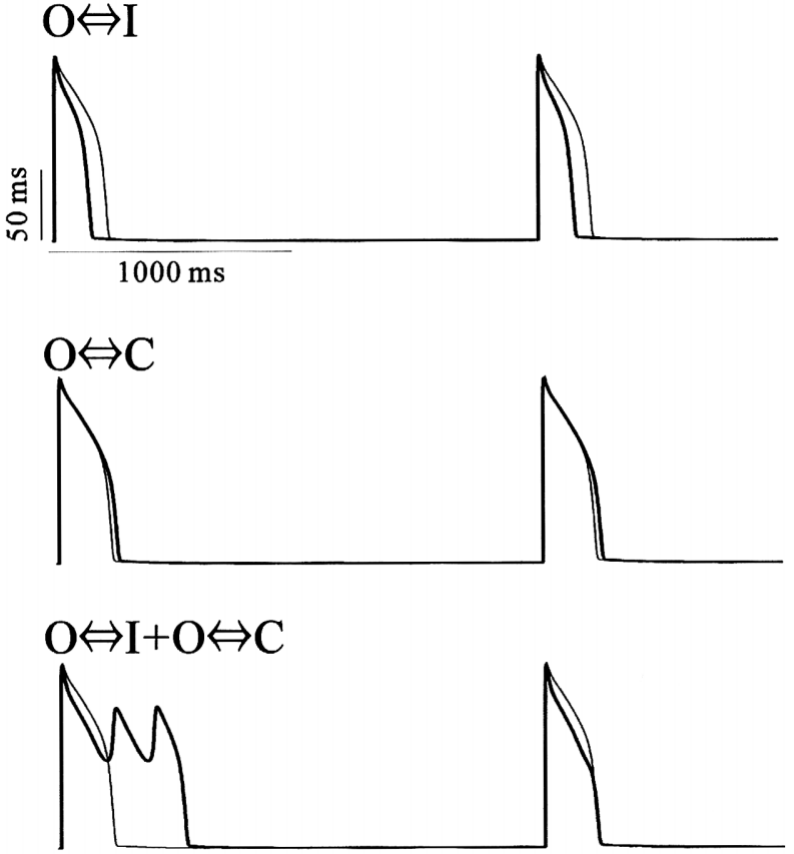

Fig 6. Individual effects of the 2 gating defects. One parameter change alone, which was associated with gain of function $(O \Leftrightarrow I)$, shortened the action potential duration (APD), but failed to cause early after-depolarizations (EAD). The accelerated deactivation $(\mathrm{C} \Leftrightarrow$ O), which was associated with decreased tail currents, did not strongly influence APD, but only the condition of the combined gating defects $(\mathrm{O} \Leftrightarrow \mathrm{I}+\mathrm{C} \Leftrightarrow \mathrm{O})$ caused EAD. Wild-type, thin lines; N588K, thick lines.

(Fig 5B). The inactivation of $\mathrm{ICa}(\mathrm{L})$ currents involves voltage-dependent and calcium-dependent processes (Fig 5C and D). In the LRd model, they are represented by 2 gates, $\mathrm{f}$ and $\mathrm{fCa}$, which are voltage- and calcium-dependent, respectively. The product of these 2 gates, $\mathrm{f} \times \mathrm{fCa}$, is an important parameter that indicates the recovery from inactivation of ICa(L) and the availability of calcium channels for subsequent reactivation? The faster recovery from inactivation at a membrane potential in the range of $\mathrm{ICa}(\mathrm{L})$ activation allows reactivation of $\mathrm{ICa}(\mathrm{L})$, which being an inward current, results in secondary membrane depolarization to generate the EAD (Fig 5D).

\section{Effects of Each Gating Defect for AP Simulations for LRd Model}

We investigated how each of the 2 gating defects would affect the myocardial model (Fig 6). Unexpectedly, changes of 1 parameter alone, which was associated with gain of function or accelerated deactivation, could change the APD but failed to cause EAD. The increased transient rate from the $\mathrm{O}$ state to the closed state, which was associated with decreased tail currents, was strongly associated with EAD of the short-QT syndrome. Only the combination of the 2 gating defects could cause EAD (Fig 6). ICa(L) reactivation also occurred only with the combination of the 2 gating defects.

\section{Discussion}

The KCNH2 potassium channel has a shaker-like tetrameric structure composed of homologous core units each containing 6 membrane-spanning segments. Co-assembly
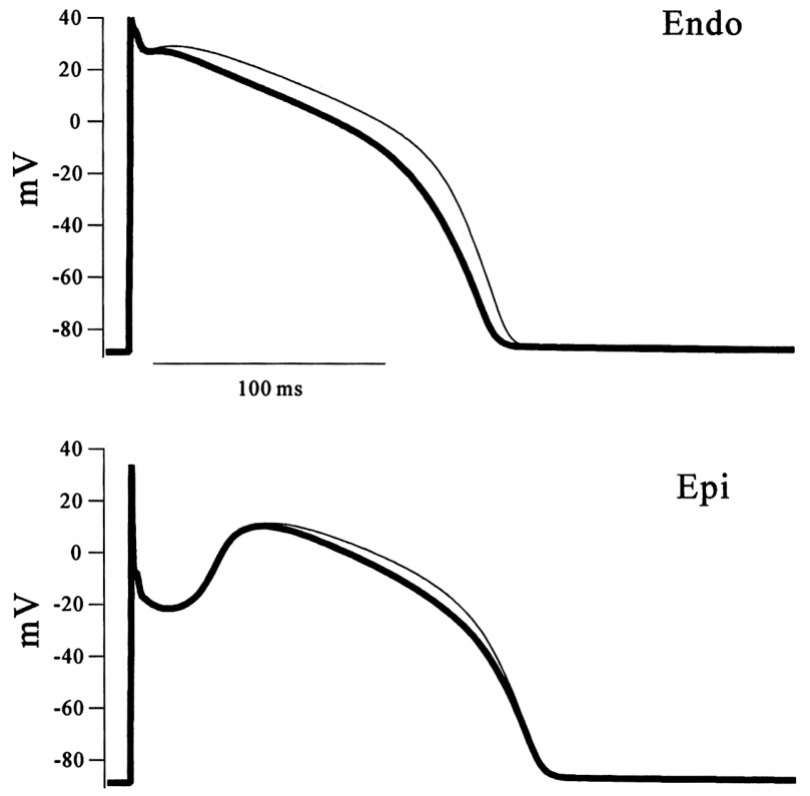

Fig 7. The contribution of transient potassium current (Ito) to phase I repolarization in epicardial (Epi) and endocardial (Endo) cells. Wildtype, thin lines; N588K, thick lines.

with the $\beta$-subunit MiRP1 (KCNE2) is required to fully reproduce the biophysical and pharmacological properties of the native $\mathrm{IKr}^{14}{ }^{4} \mathrm{KCNH} 2$ has previously been linked to a decrease in outward repolarizing current responsible for the hereditary long-QT syndrome (LQT2) ${ }^{15}$ and for acquired forms of long-QT syndrome (LQTS) $16 \mathrm{KCNH} 2$ is also the primary target of class III antiarrhythmic agents, many of which contribute to generation of an acquired form of the LQTS. Brugada et al described the first mutation associated with the short-QT syndrome? The short-QT syndrome is genetically heterogeneous. It can also be caused by mutations in the KCNQ1 gene that encodes the KVLQT1 $\mathrm{K}^{+}$ channel, which forms IKs in association with the $\beta$ subunit IsK ${ }^{17}$ and by mutations in the KCNJ 2 gene that encodes the inwardly rectifying Kir2.1 channel. ${ }^{18}$

We studied how gain of function of $\mathrm{KCNH} 2$ would cause life-threatening arrhythmia in the short-QT syndrome. The $\mathrm{N} 588 \mathrm{~K}$ mutation of $\mathrm{KCNH} 2$ was found to cause a remarkable gain of function in the IKr in short-QT syndrome? Based on the voltage-clamp experiments, the present simulation study revealed that $\mathrm{N} 588 \mathrm{~K}$ had 2 gating defects. Unexpectedly, 1 parameter change alone, which was associated with gain of function could shorten the QT intervals, but could not cause EAD by itself. The increased transition rate from the $\mathrm{O}$ state to a closed state $(\mathrm{C} 1)$, which was associated with decreased tail currents, was strongly associated with EAD of the short-QT syndrome, and only the condition with both gating defects caused EAD. The previous report showed that the accelerated deactivation is related with LQTS4. The accelerated deactivation for N588K mutant does not decrease APD, but increases APD. However, the balance between impaired inactivation and the accelerated deactivation would determine APD, and gain of function in the IKr might be a strong determinant of APD in the case of N588K. McPate et al and Cordeiro et al reported that N588K channels alter the ion selectivity 11,12 Reversal potentials for the $\mathrm{N} 588 \mathrm{~K}$ mutant are significantly higher. We also studied how the ion selectivity could affect EAD for 
N588K channels. Higher reversal potential could easily cause EAD for N588K channels with double gating defects (data not shown).

$\mathrm{ICa}(\mathrm{L})$ reactivation seems to be a universal mechanism of EAD from plateau potentials. It is well known that EAD cause ventricular tachycardia in the LQTS4,9 Prolongation of the post-pause AP plateau because of a smaller IKs or IKr and an enhanced inward $\mathrm{INaCa}$, on the background of the LQTS effects, provides time for the recovery and reactivation of $\mathrm{ICa}(\mathrm{L})$, which generates the depolarizing charge for EAD formation. However, the faster recovery from inactivation in the short-QT syndrome allows for the reactivation of $\mathrm{ICa}(\mathrm{L})$.

The group of Antzelevitch presented the first experimental evidence of the role of transmural dispersion of repolarization (TDR) in arrhythmogenesis associated with shortQT intervals in the ECG6 Pinacidil was used to mimic a gain of function of a potassium current in the canine arterially perfused wedge preparation. Pinacidile activates adenosine triphosphate-sensitive potassium currents (IKATP), leading to an abbreviation of APD!9,20 In the wedge preparation, pinacidil abbreviated the QT interval, leading to a heterogeneous abbreviation of the APD of the 3 principal cell types spanning the ventricular wall. The pinacidil model of the short-QT syndrome, although mechanistically related, is phenotypically different from the clinical syndrome caused by a gain of function of HERG.

The computer simulation is a good tool to predict APD of genetic arrhythmogenic disorders, such as the LQTS and Brugada syndrome, $, 5,7$ We could successfully reproduce these alterations of gating properties in the short-QT syndrome by changing 2 parameters in the Markov model of the KCNH2. When the IKr channels were all N588K mutants, APD of each wall layer was smaller than that of WT. The TDRmax of N588K was also smaller than WT at any BCL (data not shown). These results are opposed to the wedge models. However, Brugada et al showed that the mid- and endocardial regions of the ventricle would repolarize more rapidly than the epicardium, because currents mediated by mutated HERG channels electrontically follow the membrane potential. The presence of a notch during phase I therefore lowers the amplitude of the N588K currents in the epicardium vs endocardium and increase TDR. We tested this hypothesis by modifying the LRd model to take into account the contribution of transient potassium current (Ito) to phase I repolarization of the epicardium, according to modifications published earlier by Dumaine et al?.1 Although mutant APD for endocardial cells shorten in the presence of Ito, shortening of the APD for epicardial cells was slight, and these results lead to increase TDR (Fig 7). Cordeiro et al recently reported that the dispersion of repolarization between the Purkinje fibers and ventricles induced by $\mathrm{N} 588 \mathrm{~K}$ might create a substrate for premature re-excitation of the endocardium!1 In the short-QT syndrome, ventricular tachycardia might occur in the substrate of both EAD in M cells and the dispersion of repolarization between the Purkinje fibers and ventricles. We might need to analyze the transmural AP mapping by applying a validated optical mapping technique ? $^{2}$

Clinically, we have not clearly understood what triggers ventricular tachycardia in the short-QT syndrome unlike the LQTS 23,24 because adequate genotype-phenotype correlation data are not yet available for the short-QT syndrome. A latest report shows that shortening of the QT interval is prominent at lower heart rates 25 Therefore, we speculate that a potential trigger might occur in ventricular tachyarrhythmias at rest and during sleep in patients with a shortQT syndrome.

In summary, a part of the short-QT syndrome has been linked to gain of function mutation of $\mathrm{KCNH} 2$. Although the gain of function for $\mathrm{KCNH} 2$ causes shortened APD of the short-QT syndrome, arrhythmogenesis might be associated not only with gain of function, but also with accelerated deactivation of HERG.

\section{Limitations}

First, the simulation studies are generally simplified. However, the simulation studies lead to understand complex and interactive phenomena, and relate them to membrane ionic currents and to dynamic changes in the intracellular ionic environment? Second, the simulation studies were conducted in isolated cell models. The ionic mechanism in the single cell helps us to understand the basis of its behavior in the multicellular tissue with complex interactions. In the intact myocardium, cells are interconnected through gap-junctions and we need to study its ionic mechanism in the multicellular tissue level? ${ }^{26}$

\section{Acknowledgment}

We thank Y Rudy, CE Clancy and their colleagues for the simulation programs which are open to the public. We also thank Lippincott Williams \& Wilkins for allowing reproduction of their figures.

\section{References}

1. Gussak I, Brugada P, Brugada J, Wright RS, Kopecky SL, Chaitman BR, et al. Idiopathic short QT interval: A new clinical syndrome? Cardiology 2000; 94: 99-102.

2. Gaita F, Giustetto C, Bianchi F, Wolpert C, Schimpf R, Riccardi R, et al. Short QT syndrome: A familial cause of sudden death. Circulation 2003; 108: $965-970$.

3. Brugada R, Hong K, Dumaine R, Cordeiro J, Gaita F, Borqqrefe M, et al. Sudden death associated with short-QT syndrome linked to mutations in HERG. Circulation 2004; 109: 30-35.

4. Clancy CE, Rudy Y. Cellular consequences of HERG mutations in the long QT syndrome: Precursors to sudden cardiac death. Cardiovasc Res 2001; 50: 301-313.

5. Clancy CE, Rudy Y. $\mathrm{Na}^{+}$channel mutation that causes both Brugada and long-QT syndrome phenotypes. Circulation 2002; 105: $1208-$ 1213.

6. Extramiana F, Antzelevitch C. Amplified transmural dispersion of repolarization as the basis for arrhythmogenesis in a canine ventricular-wedge model of short-QT syndrome. Circulation 2004; 110: $3661-3666$.

7. Viswanathan PC, Rudy Y. Pause induced early after depolarization in the long QT syndrome: A simulation study. Cardiovasc Res 1999; 42: $530-542$.

8. Haraguchi Y, Yoshinaga M, Sarantuya J, Shimago A, Nishi J, Kono $\mathrm{Y}$, et al. Interval representative of transmural dispersion of repolarization in children and young adolescents with long QT syndrome. Circ J 2005; 69: 78-82.

9. Viswanathan PC, Shaw RM, Rudy Y. Effects of IKr and IKr heterogeneity on action potential duration and its rate dependence: A simulation study. Circulation 1999; 99: 2466-2474.

10. Faber GM. Development of the Luo-Rudy (LR) model of the mammalian cardiac ventricular cell. http://www.cwru.edu/med/CBRTC/ LRdOnline/development.htm, 2001.

11. Cordeiro JM, Brugada R, Wu YS, Hong K, Dumaine R. Modulation of IKr inactivation by mutation N588K in KCNH2: A link to arrhythmogenesis in short-QT syndrome. Cardiovasc Res 2005; 67: $498-$ 509 .

12. McPate MJ, Duncan RS, Milnes JT, Witchel HJ, Hancox JC. The N588K-HERG K+ channel mutation in the 'short QT syndrome': Mechanism of gain-in-function determined at $37^{\circ}$. Biochem Biophys Res Commun 2005; 334: 441-449.

13. Zhou Z, Gong Q, Ye B, Fan Z, Makielski JC, Robertson GA, et al. Properties of HERG channels stably expressed in HEK293 cells studied at physiological temperature. Biophys J 1998; 74: 230-241.

14. Ficker E, Jarolimek W, Kiehn J, Baumann A, Brown AM. Molecular 
determinants of dofetilide block of HERG $\mathrm{K}^{+}$channels. Circ Res 1998; 82: 386-395.

15. Curran ME, Splawski I, Timothy KW, Vincent GM, Green ED, Keating MT. A molecular basis for cardiac arrhythmia: HERG mutations cause long QT syndrome. Cell 1995; 80: 795-803.

16. Roden DM. Pharmacogenetics and drug-induced arrhythmias. Cardiovasc Res 2001; 50: 224-231.

17. Bellocq C, van Ginneken AC, Bezzina CR, Alders M, Escande D, Mannens MM, et al. Mutation in the KCNQ1 gene leading to the short QT-interval syndrome. Circulation 2004; 109: 2394-2397.

18. Priori SG, Pandit SV, Rivolta I, Berenfeld O, Ronchetti E, Dhamoon A, et al. A novel form of short QT syndrome (SQT3) is caused by a mutation in the KCNJ2 gene. Circ Res 2005; 96: 800-807.

19. Arena JP, Kass RS. Activation of ATP-sensitive K channels in heart cells by pinacidil: Dependence on ATP. Am J Physiol 1989; 257: H2092-H2096.

20. Tseng GN, Hoffman BF. Actions of pinacidil on membrane currents in canine ventricular myocytes and their modulation by intracellular ATP and cAMP. Pflugers Arch 1990; 415: 414-424.
21. Dumaine R, Towbin JA, Brugada P, Vatta M, Nesterenko DV, Nesterenko VV, et al. Ionic mechanisms responsible for the electrocardiographic phenotype of the Brugada syndrome are temperature dependent. Circ Res 1999; 85: 803-809.

22. Akar FG, Yan GX, Antzelevitch C, Rosenbaum DS. Unique topographical distribution of $\mathrm{M}$ cells underlies reentrant mechanism of torsades de pointes in the long-QT syndrome. Circulation 2002; 105: $1247-1253$

23. Urao N, Shiraishi H, Ishibashi K, Hyogo M, Tsukamoto M, Keira N, et al. Idiopathic long QT syndrome with early after depolarization induced by epinephrine: A case report. Circ J 2004; 68: 587-591.

24. Yoshinaga M, Nagashima M, Shibata T, Niimura I, Kitada M, Yasuda T, et al. Who is at risk for cardiac events in young patients with long QT syndrome? Circ J 2003; 67: 1007-1012.

25. Schimpf R, Wolpert C, Gaita F, Giustetto C, Borggrefe M. Short QT syndrome. Cardiovasc Res 2005; 67: 357-366.

26. Viswanathan PC, Shaw RM, Rudy Y. Effects of IKr and IKs heterogeneity on action potential duration and its rate dependence: A simulation study. Circulation 1999; 99: 2466-2474. 